\title{
ALTITUDE TRAJECTORY CONTROL OF AN UNMANNED AIRSHIP USING INCREMENTAL NONLINEAR DYNAMIC INVERSION
}

\author{
Ali Mansur ${ }^{1}$, Eric Lanteigne ${ }^{2}$ \\ Mechanical engineering Department, University of Ottawa, Ottawa, Canada \\ amans070@uottawa.ca, eric.lanteigne@uottawa.ca
}

\begin{abstract}
This paper presents the application of the incremental nonlinear dynamic inversion (INDI) control approach for the trajectory tracking of an unmanned airship. The proposed vehicle architecture is composed of two main components: a helium envelop with a rigid keel and moving gondola capable of travelling along the keel. This motion significantly alters the location of the centre of gravity and thus the pitch of the airship. The dynamic equations of the airship are derived using the Newton-Euler formulation, and the model was implemented and simulated in Matlab/Simulink to illustrate the effectiveness of the designed controller for translational and longitudinal motions in the presence of wind disturbances.
\end{abstract}

Keywords-component; unmanned airship; trajectory tracking; incremental nonlinear dynamic inversion

\section{INTRODUCTION}

Incremental nonlinear dynamic inversion (INDI) control is a variation on the nonlinear dynamic inversion (NDI). The NDI is a nonlinear control method which eliminates the system nonlinearity by means of feedback and results into partly or entirely linearized closed-loop system dynamics, where conventional linear control techniques can then be applied. The INDI method retains the high-performance characteristics of NDI while reducing model dependency, increasing robustness and reducing computational complexity [1-2]. The INDI only requires knowledge of the system kinematics and the actuator dynamics. The dynamic model is replaced with measurements of the system dynamics [3]. The INDI controller solves the incremental form of equations of motion using acceleration feedback and generates a control law substantially reducing the controller's dependence on complex, and sometimes inaccurate, models such as those describing the aerodynamic phenomena in flight dynamics. Feedback of angular accelerations eliminates the sensitivity to model mismatch, greatly increasing the performance of the system compared with conventional nonlinear dynamic inversion [4]. The incremental nonlinear dynamic inversion method has been successfully used to control various aerospace systems and shown desirable robust performance to aerodynamic model uncertainties [4-8]. The concept of INDI was first suggested in [9]. They examined the behaviour of NDI control law on the VAAC Harrier. During flight test a poor quality angular accelerations signals were observed and filtering and differentiating the angular rates led to oscillatory closed-loop behaviour. They suggested that the preferred solution would be to use an independent angular acceleration signals. An extension of Smith's approach to reformulate the dynamic inversion to be less dependent on the onboard model was developed in [10]. They chose two different sources to get independent data of the state acceleration vector: linear accelerometers and numerically differentiated angular rates. Angular acceleration data was derived from a unique implementation of linear accelerometer measurements, in addition to the differentiated angular rates. Recently, the INDI methodology has been studied at Delft University of Technology [4]. INDI was adopted to control the attitude of a fixed-wing aircraft and, when compared to NDI, demonstrated improved robustness to model uncertainties. More recently INDI control theory was used to achieve a complete quadrotor controller in [11]. INDI showed improvements with the control of a time varying quadrotor model. The INDI controller was able to maintain the quadrotor trajectory while performing some degree of aggressive manoeuvres.

INDI control was applied to the lateral control of an under actuated airship with uncertain dynamics model [5]. The stability and robustness of the proposed sensor-based control solution was illustrated with representative simulation results, including wind disturbances and a path following loop. The INDI controller performance was evaluated by adapting the DRONI's geometry with four-propellers configuration of the AS800 of [12]. The controller was successfully able to follow the path with a maximum error of $10 \mathrm{~m}$ in the presence of wind disturbances.

The derivations of INDI control law in literature are based on the time scale separation principle, which considers that the controls can change significantly faster than the states when the sampling frequency is high [4-8]. The time scale separation principle uses the fact that the time constants of the inner and outer loops are different and hence the control laws for inner and outer loops can be designed independently. The closedloop system stability of a general linear system controlled by INDI was investigated in [13]. The analytical stability analysis showed that implementing discrete-time INDI with a smaller sampling time resulted in larger stability margins regarding system characteristics and controller gains. 
The reduction of the noise is essential in the development of an INDI controller. Several approaches were taken into consideration to address this issue. A first order filter used in [14] to reduce the effect of noise and time-delays. Veld proposed three filters to compensate for sensor noise: a firstorder low-pass filter, a second-order low-pass filter and two low-pass filters [1]. A linear predictive filter was used to predict the angular accelerations, solve the time delay and angular acceleration availability problem [4]. Other methods include improvements in the indirect measurement of angular accelerations by using Recurrent Neural Networks [15].

INDI is also sensitive to the system sampling frequency [16-17]. It was observed that the system becomes unstable when the sampling frequency falls below $50 \mathrm{~Hz}$ leading to a higher overshoot, noticeable ringing and longer settling time [16]. High sampling rates can compensate for model uncertainties and simplifications. The results in [6] showed that the INDI controller was robust to uncertainties in the control matrix, with a sampling rate above $50 \mathrm{~Hz}$.

Signal delay is third main challenges in INDI control. Examples of delays include the differentiation of angular rates for the computation of the angular accelerations and the filters needed to decrease noise in the linear acceleration measurements. This is particularly important in the former as differentiation amplifies the sensor noise [18]. A solution to prevent performance degradation due to the time delay are to synchronize the time delays of all signals by applying the same filter to the angular rate measurements [8]. In addition, Pseudo control hedging can be used to reduces the magnitude of the commanded signals to a level achievable by the saturated controller [13].

In this paper, the INDI methodology is applied to the trajectory tracking of the vehicle architecture shown in Fig. 1. The controller developed in [5] was modified for longitudinal motion, and the effect of the moving gondola for rapid altitude changes for ascending or landing an airship was studied.

\section{AIRSHIP ARCHITECTURE AND NONLINEAR DYNAMIC MODEL}

\section{A. Airship Architecture}

The vehicle architecture is shown in Fig. 1. It is comprised of a semi-rigid helium envelope and a moving gondola. The gondola moves on a rail fixed to the keel of the helium envelope. The rail is strait along the mid-section of the vehicle and circular along the bow. This allows the thruster to remain in line with the centre of volume (CV) of the airship at all times. This vehicle configuration provides an alternative solution to over-actuation and ballonets for the rapid altitude changes required when landing or avoiding obstacles. The physical properties of the airship are listed in Table 1. The airship is $3 \mathrm{~m}$ long and the gondola can travel along the keel from $s_{g}=-0.81 \mathrm{~m}$ to $s_{g}=0.96 \mathrm{~m}$ along the straight section and from $s_{g}=0.96 \mathrm{~m}$ to $s_{g}=1.40 \mathrm{~m}$ along the circular section, where $s_{g}=0 \mathrm{~m}$ is defined as the gondola position directly below the $\mathrm{CV}$.

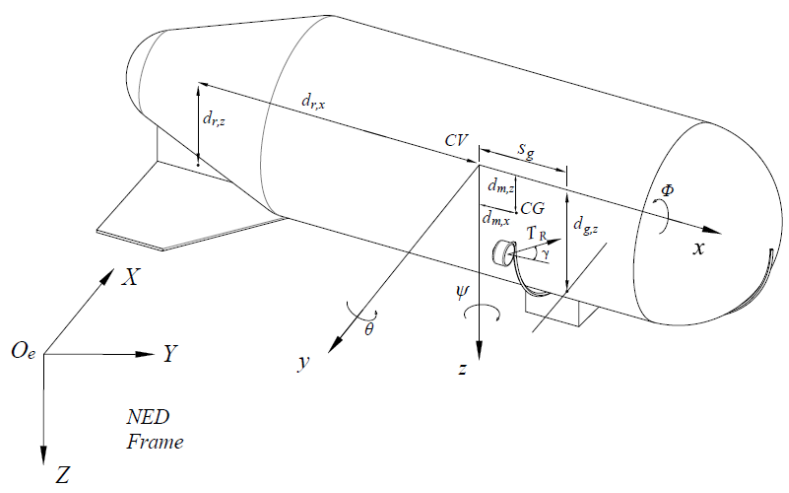

Figure 1. Body axis convention and inertia axis of the airship

\section{B. Nonlinear Dynamic Model}

The nonlinear dynamic model for the proposed architecture was derived using the Newton-Euler method similar to [19] and [20]. Two frames of reference were defined to describe the airship motion; the inertial North-East-Down (NED) reference axis system fixed on the earth and the body axis system fixed to the airship CV. The orientation of the body axis system is represented by Euler angles (roll $\phi$ pitch $\theta$ and yaw $\psi$ ).

The model's reference system is shown in Fig. 1 and its equation of motion is defined as,

$$
\mathbf{M} \ddot{\mathbf{x}}_{\mathbf{v}}+\mathbf{D}\left(\dot{\mathbf{x}}_{\mathbf{v}}\right)=\mathbf{E}+\mathbf{U}
$$

where, $\mathbf{M}$ is the $6 \times 6$ mass matrix, $\mathbf{D}$ is the $6 \times 1$ dynamics vector, $\mathbf{E}$ is the $6 \times 1$ model uncertainty vector, $\mathbf{x}_{\mathrm{v}}$ is the $6 \times 1$ state vector, the vector of external forces and moments $\mathbf{U}$ is further defined as,

$$
\mathbf{U}=\mathbf{A}\left(\dot{\mathbf{x}}_{\mathbf{v}}\right)+\mathbf{G}\left(\boldsymbol{R}_{1}\right)+\mathbf{F}_{u},
$$

where, $\mathbf{A}$ is the $6 \times 1$ aerodynamic vector, $\mathbf{G}$ is the $6 \times 1$ gravitational and buoyancy vector, $\boldsymbol{R}_{l}$ is the directional cosine matrix, and $\mathbf{F}_{\mathrm{u}}$ is the $6 \mathrm{x} 1$ propulsive force vector. The complete derivation of the terms in (1) and (2) are provided in [20]. The state convention $\mathbf{x}$ is written as,

$$
\begin{gathered}
\mathbf{x}=\left[\begin{array}{llllll}
x & y & z & \phi & \theta & \psi
\end{array}\right]^{T}, \quad \dot{\mathbf{x}}=\left[\begin{array}{ll}
V & \omega
\end{array}\right]^{T}, \\
V=\left[\begin{array}{lll}
\dot{x} & \dot{y} & \dot{z}
\end{array}\right]^{T}, \quad \omega=\left[\begin{array}{lll}
\dot{\phi} & \dot{\theta} & \dot{\psi}
\end{array}\right]^{T},
\end{gathered}
$$

where $V$ is vector of linear velocities and $\omega$ is angular velocities of the vehicle defined in the moving frame of reference located at the $\mathrm{CV}$ of the helium envelope.

$$
\boldsymbol{R}_{I}=\left[\begin{array}{ccc}
\mathrm{c} \theta \mathrm{c} \psi & \mathrm{s} \phi \mathrm{s} \theta \mathrm{c} \psi-\mathrm{c} \phi \mathrm{s} \psi & \mathrm{c} \phi \mathrm{s} \theta \mathrm{c} \psi+\mathrm{s} \phi \mathrm{s} \psi \\
\mathrm{c} \theta \mathrm{s} \psi & \mathrm{s} \phi \mathrm{s} \theta \mathrm{s} \psi+\mathrm{c} \phi \mathrm{c} \psi & \mathrm{c} \phi \mathrm{s} \theta \mathrm{s} \psi-\mathrm{s} \phi \mathrm{c} \psi \\
-\mathrm{s} \theta & \mathrm{s} \phi \mathrm{c} \theta & \mathrm{c} \phi \mathrm{c} \theta
\end{array}\right]
$$

The rotation matrix $\boldsymbol{R}_{\boldsymbol{I}}$ (also known as the 123 or roll-pitch-yaw Euler sequence), 
The mass of the airship $(m)$ is equal to the sum of the mass of the gondola $\left(m_{g}\right)$, the rail $\left(m_{r}\right)$, the envelope $\left(m_{e}\right)$, the motors $\left(m_{m}\right)$ and the fins $\left(m_{f}\right)$. The centre of mass $(\mathrm{CM})$ of the airship is calculated as,

$$
\begin{aligned}
& d_{m, x}=\frac{m_{g} \times s_{g}+\left(2 \times m_{m} \times x_{m}\right)}{m} \\
& d_{m, z}=\frac{m_{g} \times z_{g}+m_{r} \times L_{r}+m_{f} \times L_{f}}{m}
\end{aligned}
$$

where $d_{m, x}$ and $d_{m, z}$ are the vertical distances between CM and $\mathrm{CV}$ along $\mathrm{x}$ and $\mathrm{z}$-axes respectively, and

$$
\begin{gathered}
\left.\begin{array}{c}
z_{g}=d_{p, z} \\
x_{m}=s_{g}
\end{array}\right\} \quad-0.81 \leq s_{g} \leq 0.96 \\
\left.\begin{array}{c}
z_{g}=d_{p, z} \times \cos \zeta \\
x_{m}=s_{g}-\left(d_{p, z} \times \sin \zeta\right)
\end{array}\right\} \quad s_{g}>0.96
\end{gathered}
$$

where $z_{g}$ is the distance between the $\mathrm{CV}$ to the $\mathrm{CM}$ of the gondola along $\mathrm{z}, x_{m}$ is distance between the $\mathrm{CV}$ to $\mathrm{CM}$ of the propeller along $\mathrm{x}, \zeta$ is the angle between the link and $\mathrm{z}$ axis when the gondola is in the curved link. $L_{f}$ and $L_{r}$ are the distance between the $\mathrm{CV}$ to the $\mathrm{CM}$ of the rail and fin along $\mathrm{z}$ respectively. The masses and the inertias of the fixed components as indicated by the subscript $f$ represent those of the envelope and rail and were found based on the dimensions of the prototype solid model.

All other variables and coefficients are summarized in [20], and including references to where they can be determined for any airship platform using a combination of semi empirical models and geometric functions.

\section{Kinematics}

Vehicle kinematics were applied in tandem to determine the velocity and positional changes and how they translate to the earth reference frame. Coordinates and trajectories in the earth reference frame can be determined from the following equation,

$$
\dot{\mathbf{x}}_{\mathrm{g}}=\mathbf{J} \dot{\mathbf{x}}_{\mathrm{v}}+\boldsymbol{v}_{\mathrm{w}}=\left[\begin{array}{cc}
\boldsymbol{R}_{1} & 0_{3 x 3} \\
0_{3 x 3} & \boldsymbol{R}_{2}
\end{array}\right] \dot{\mathbf{x}}_{\mathrm{v}}+\boldsymbol{v}_{\mathrm{w}}
$$

where $v_{w}$ is the total wind (steady + gusts) in a $6 \times 1$ vector and, $\boldsymbol{R}_{2}$ is the rotation matrix described by

$$
\boldsymbol{R}_{2}=\left[\begin{array}{ccc}
1 & \sin \phi \tan \theta & \cos \phi \tan \theta \\
0 & \cos \phi & -\sin \phi \\
0 & \sin \phi \cos \theta & \cos \phi \sec \theta
\end{array}\right]
$$

\section{INCREMENTAL NONLINEAR DYNAMICS INVERSION APPROACH}

Instead of computing the total control input directly, the INDI methodology calculates the increment of the control input for every sample time based on the system states at the previous time step. This concept has been used for the purpose of coping with nonlinear control derivatives, and to reduce the impact of model mismatch [4]. Given a general nonlinear system:

$$
\dot{\mathbf{x}}=\mathbf{f}(\mathbf{x})+\mathbf{G}(\mathbf{x}) \mathbf{u}
$$

where $\mathbf{x} \in \mathbb{R}^{n}$ is the state vector, $\mathbf{u} \in \mathbb{R}^{m}$ is the input, $\mathbf{G} \in \mathbb{R}^{n \times m}$ is the control effectiveness matrix, the Taylor series expansion provides the following first-order approximation of $\dot{\mathbf{x}}$ at the previous state and control $\mathbf{x}_{\mathbf{0}}, \mathbf{u}_{\mathbf{0}}$ respectively,

$$
\begin{aligned}
\dot{\mathbf{x}}= & f\left(\mathbf{x}_{\mathbf{0}}\right)+\mathrm{G}\left(\mathbf{x}_{\mathbf{0}}\right) \mathbf{u}_{\mathbf{0}}+\left.\frac{\partial}{\partial \mathbf{x}}[\mathrm{f}(\mathbf{x})+\mathrm{G}(\mathbf{x}) \mathbf{u}]\right|_{\substack{x=\mathbf{x}_{0} \\
\mathbf{u}=\mathbf{u}_{0}}}\left(\mathbf{x}-\mathbf{x}_{\mathbf{0}}\right) \\
& +\left.\frac{\partial}{\partial \mathrm{u}}[\mathrm{G}(\mathbf{x}) \mathbf{u}]\right|_{\substack{x=\mathbf{x}_{0} \\
\mathrm{u}=\mathrm{u}_{0}}}\left(\mathbf{u}-\mathbf{u}_{\mathbf{0}}\right)+\text { H.O.T }
\end{aligned}
$$

Assuming a high sampling frequency, the changes of state variation $\mathbf{x} \approx \mathbf{x}_{\mathbf{0}}$ of equation (8) and (H.O.T) the higher order terms can be neglected. The dynamics of the previous state $\dot{\mathbf{x}}_{\mathbf{0}}$ are:

$$
\begin{gathered}
\dot{\mathbf{x}}_{\mathbf{0}}=\mathrm{f}\left(\mathbf{x}_{\mathbf{0}}\right)+\mathrm{G}\left(\mathbf{x}_{\mathbf{0}}\right) \mathbf{u}_{\mathbf{0}} \\
H_{u}=\left.\frac{\partial}{\partial \mathrm{u}}[\mathrm{G}(\mathbf{x}) \mathbf{u}]\right|_{\substack{\mathrm{x}=\mathrm{x}_{0} \\
\mathrm{u}=\mathrm{u}_{0}}}
\end{gathered}
$$

where $H_{u}$ is the partial derivative of $[\mathrm{G}(\mathbf{x}) \mathbf{u}]$ at $\mathbf{x}=\mathbf{x}_{\mathbf{0}}$ and $\mathbf{u}=\mathbf{u}_{\mathbf{0}}$. The incremental form of the dynamic equation is thus simplified as,

$$
\dot{\mathbf{x}}=\dot{\mathbf{x}}_{\mathbf{0}}+H_{u}\left(\mathbf{u}-\mathbf{u}_{\mathbf{0}}\right)
$$

Equation (10) is a linear approximation of the equation of motion around $\mathbf{u}_{\mathbf{0}}$ and $\mathbf{x}_{\mathbf{0}}$ for small time increments, describing the changes in accelerations as a function of control increments. By setting $\dot{\mathbf{x}}$ as the virtual control variable $v_{r}$, the controller can then be designed in the incremental form as,

$$
\mathbf{u}=\mathbf{u}_{0}+H_{u}^{-1}\left(v_{r}-\dot{\mathbf{x}}_{0}\right)
$$

where $\dot{\mathbf{x}}_{\mathbf{0}}$ is assumed to be measurable and the input control matrix $H_{u}$ is assumed to be invertible. The total input can be obtained by adding the previous input $\mathbf{u}_{\mathbf{0}}$ to the calculated increment

$$
\mathbf{u}=\mathbf{u}_{\mathbf{0}}+\Delta \mathbf{u}
$$

It is important to notice that this implicit control law is not entirely independent on the model since changes in $f(\mathbf{x})$ are reflected in measurements of $\dot{\mathbf{x}}_{\mathbf{0}}$, improving robustness against model uncertainties contained therein. Therefore, this implicit control law is dependent on accurate measurements of the accelerations and the control input.

The acceleration reference for the INDI controller $\boldsymbol{v}_{\boldsymbol{r}}$ is obtained as follow. The position error is calculated from the difference between the reference trajectory and the feedback of the vehicle's position, and then the desired velocity towards a goal position is calculated by a proportional controller by multiplying the position error with a gain.

$$
\dot{x}_{d}=\left(x_{d}-x\right) \mathrm{k}_{\mathrm{px}}
$$

Finally, the velocity error, resulting from subtracting the desired velocity from the feedback velocity of the vehicle, is 
then multiplied with a gain to calculate the acceleration reference.

$$
v_{r}=\left(\dot{x}_{d}-\dot{x}\right) \mathrm{k}_{\mathrm{pv}}
$$

where $\mathrm{k}_{\mathrm{px}}, \mathrm{k}_{\mathrm{pv}}$ are the proportional gains of the position and velocity vectors, respectively. The proportional control is used to correct for errors in position and the velocity and provides the acceleration reference for the INDI controller. Then equation (11) becomes,

$$
\mathbf{u}=\mathbf{u}_{\mathbf{0}}+H_{u}^{-1}\left(\left[\left(\boldsymbol{x}_{\boldsymbol{d}}-\boldsymbol{x}\right) \mathrm{k}_{\mathrm{px}}-\dot{\boldsymbol{x}}\right] \mathrm{k}_{\mathrm{pv}}-\dot{\mathbf{x}}_{\mathbf{0}}\right)
$$

The equation shows that the dependency of the closed-loop system on the accurate knowledge of the airship model is largely decreased, improving robustness against model uncertainties. Therefore, the changes in $\mathrm{f}(\mathbf{x})$ are reflected in $\dot{\mathbf{x}}_{\mathbf{0}}$, and the control mainly required the measurements of $\dot{\mathbf{x}}_{\mathbf{0}}$ and $\mathbf{u}_{\mathbf{0}}$, making this control approach more dependent on the sensor measurements.

Different filtering approaches have been used to solve the problem of indirect measurement of the accelerations. Usually, the acceleration in $\dot{\mathbf{x}}_{\mathbf{0}}$ is not measured directly and the sensors are not widely available, especially for small-unmanned aircrafts [11]. According to [8] the acceleration $\dot{\mathbf{x}}_{\mathbf{0}}$ can be calculated by passing the velocity component of $\dot{\boldsymbol{x}}$ through a second order low pass filter.

\section{A. Translational Control Loop}

In translational control loop, the vehicle's flight position is controlled by the vectored thrust $\mathbf{F}_{u}$ to track the reference trajectory. The vectored thrust is defined as,

$$
\mathbf{F}_{u}=\left[\begin{array}{c}
F_{x} \\
F_{z}
\end{array}\right]=\left[\begin{array}{c}
T_{R} \cos \gamma_{R}+T_{L} \cos \gamma_{L} \\
-T_{R} \sin \gamma_{R}+T_{L} \sin \gamma_{L}
\end{array}\right]
$$

where $F_{x}, F_{z}$ are the components of the thrust in body fixed frame in the $\mathrm{x}$ and $\mathrm{z}$ axes, respectively. $T_{R}$ and $T_{L}$ are the right and left propeller thrust, and $\gamma_{R}$ and $\gamma_{L}$ the right and left angles of the thrusters. The vectored thrust is considered as the control variable $\mathbf{u}_{F}$ for the INDI controller.

$$
\mathbf{u}_{F}=\left[\begin{array}{l}
F_{x} \\
F_{z}
\end{array}\right], g_{1}(\mathrm{x})=\left[\begin{array}{ll}
1 & 0 \\
0 & 1
\end{array}\right], \quad \text { thus } \quad \boldsymbol{F}_{u}=g_{1}(\mathbf{x}) \mathbf{u}_{F}
$$

Equation (1) can be rewritten into the following control affine form of (7) for the linear motion,

and

$$
\begin{gathered}
\ddot{\mathbf{x}}_{\mathrm{v}}=\mathbf{M}^{-1}\left[\mathbf{A}\left(\dot{\mathbf{x}}_{\mathrm{v}}\right)+\mathbf{G}\left(\boldsymbol{R}_{1}\right)-\mathbf{D}\left(\dot{\mathbf{x}}_{\mathrm{v}}\right)\right]+\mathbf{M}^{-1} \boldsymbol{F}_{u} \\
\mathrm{f}\left(\mathbf{x}_{\mathrm{v}}\right)=\mathbf{M}^{-1}\left[\mathbf{A}\left(\dot{\mathbf{x}}_{\mathrm{v}}\right)+\mathbf{G}\left(\boldsymbol{R}_{1}\right)-\mathbf{D}\left(\dot{\mathbf{x}}_{\mathrm{v}}\right)\right], \quad H_{u 1}=\mathbf{M}^{-1} g_{1}(\mathbf{x})
\end{gathered}
$$

TABLE I. AIRSHIP PHYSICAL PROPERTIES

\begin{tabular}{|llcc|}
\hline Term & Value & Term & Value \\
\hline$m$ & $1.66 \mathrm{~kg}$ & $d_{p, z}$ & $0.533 \mathrm{~m}$ \\
$V$ & $1.422 \mathrm{~m}^{3}$ & $I_{x}$ & $0.1 \mathrm{~m}^{2} . \mathrm{kg}$ \\
$D$ & $0.86 \mathrm{~m}$ & $I_{y}$ & $0.3 \mathrm{~m}^{2} . \mathrm{kg}$ \\
$d_{g, z}$ & $0.533 \mathrm{~m}$ & $I_{z}$ & $0.3 \mathrm{~m}^{2} . \mathrm{kg}$ \\
$d_{p, y}$ & $0.533 \mathrm{~m}$ & & \\
\hline
\end{tabular}

The incremental form of the translational control is,

$$
\mathbf{u}_{F}=\mathbf{u}_{F O}+\Delta \mathbf{u}_{F}
$$

where $\mathbf{u}_{F 0}$ is the previous input. The airship's flight path control can be calculated by using the translational equation of motion and (11). The subscript (v) represents the linear equations of motion used to distinguish between the linear and rotational equations. The linear acceleration $\dot{\mathbf{x}}_{\mathbf{v} \mathbf{0}}$ can be measured by onboard sensors or by passing the velocity $\dot{\boldsymbol{x}}$ through a second order low pass filter as used in this study. The term $\mathrm{f}\left(\mathbf{x}_{\mathbf{0}}\right)$ was cancelled in (9) which reduces the controller's dependence on the model accuracy. The linear acceleration reference $v_{r}$ is obtained from (12), as a state error feedback with constant gains.

\section{B. Altitude Control Loop}

The objective of this control loop is to control the pitch angle by controlling the gondola's position along the length of the airship keel. The angular rate is controlled by the moments of gravitational and buoyancy vector $\mathbf{G}$.

$$
\mathbf{G}_{M}=\left[\begin{array}{lll}
G_{\phi} & G_{\theta} & G_{\psi}
\end{array}\right]^{T}
$$

where $\mathbf{G}_{M}$ is the moment vector of gravitational and buoyancy, $G_{\phi}$ and $G_{\psi}$ are the moment components of gravitational and buoyancy vector around the $\mathrm{x}$ axis and $\mathrm{z}$ axis and are considered here equal to zero in order to study the effects of the gondola position on the altitude control in the $\mathrm{X}-\mathrm{Z}$ plane. $G_{\theta}$ is the moment component of gravitational and buoyancy vector around the $\mathrm{y}$ axis. The gravitational and buoyancy forces applied to the vehicle must be premultiplied by a rotation matrix to convert them to the body reference frame. After some manipulations, the final format of $G_{\theta}$ is,

$$
\begin{aligned}
G_{\theta}= & {\left[R(3,1) \times g\left(d_{m, x}\left(m-m_{g}\right)+m_{g} d_{m, z}\right)\right] } \\
& -\left[R(3,3) \times g\left(d_{m, x}\left(m-m_{g}\right)+m_{g} s_{g}\right)\right]
\end{aligned}
$$

where $d_{m, x}$ is a function of $s_{g}$. The input $s_{g}$ can be calculated by substituting $d_{m, x}$ in (17) and solving for $s_{g}$, the equation (17) can be rewritten as,

$$
G_{\theta}=g_{2}(\mathbf{x}) s_{g}
$$

where $g_{2}(\mathbf{x})$ contains the terms directly related with $s_{g}$. The equation shows that the movement of the moving mass $s_{g}$ is the primary factor affecting the pitching moment. In this loop, the control variable is the gondola's position $s_{g}$.

Equation (1) can be rewritten into the following control affine form equation (7) for the angular motion,

and

$$
\begin{aligned}
\ddot{\mathbf{x}}_{\omega} & =\mathbf{M}^{-1}\left[\mathbf{A}\left(\dot{\mathbf{x}}_{\omega}\right)+\mathbf{F}_{u}-\mathbf{D}\left(\dot{\mathbf{x}}_{\omega}\right)\right]+\mathbf{M}^{-1} \mathbf{G}\left(\boldsymbol{R}_{1}\right) \\
\mathrm{f}\left(\mathbf{x}_{\omega}\right) & =\mathbf{M}^{-1}\left[\mathbf{A}\left(\dot{\mathbf{x}}_{\omega}\right)+\mathbf{F}_{u}-\mathbf{D}\left(\dot{\mathbf{x}}_{\omega}\right)\right], \quad H_{u 2}=\mathbf{M}^{-1} g_{2}(\mathbf{x})
\end{aligned}
$$

The incremental form of the longitudinal control is, 


$$
s_{g}=s_{g 0}+\Delta s_{g}
$$

The airship's pitch angle control can be calculated by using the rotational equation of motion and (11). The subscript $(\omega)$ represents the rotational equations of motion used to distinguish between the linear and rotational equations. The angular acceleration $\dot{\mathbf{x}}_{\omega 0}$ can be measured by onboard sensors or by passing the angular velocity $\dot{\omega}$ through a second order low pass filter. The measured accelerations in both loops were filtered by a second order low pass filter to keep all signals synchronized in control equations and to prevent performance degradation of the controller due to the time delay [8]. The angular acceleration reference $\boldsymbol{v}_{\boldsymbol{r}}$ is obtained from (12), as a state error feedback with constant gains.

\section{NUMERICAL SIMULATION}

Numerical simulations were carried out to illustrate the altitude tracking performance of the controller altitude. The dynamic simulation was developed in the Matlab/Simulink environment. Rate limiters and output saturation were added to the closed-loop system to respect the geometry, and actuator and thruster capability and saturation. A maximum gondola repositioning rate of $\dot{s}_{g}=0.3 \mathrm{~m} / \mathrm{s}$ was adopted based on openloop hardware tests on the gearmotors driving the gondola. The propeller thrust was limited to $-0.26 \mathrm{~N}<\mathrm{T}<2.6 \mathrm{~N}$ based on thruster static bench testing prototype. The acceleration $\dot{\mathbf{x}}_{0}$ calculated by passing the velocity $\dot{\boldsymbol{x}}$ through a second order low pass filter with natural frequency $\omega_{n}=25$ and damping $\zeta_{n}=0.7 \mathrm{rad} / \mathrm{s}$.

The controller gains were selected heuristically to obtain a desired closed loop response. The position gains and the velocity gains of the translational control are, $\left(K_{x p}=3, K_{z p}=2\right.$, $\left.K_{x v}=5, K_{z v}=6\right)$ and the longitudinal controller gains. ( $K_{\theta}=8$, $\left.K_{\dot{\theta}}=2\right)$. In the simulation process, the initial position of $\mathrm{CV}$ of the airship is $\left(\mathrm{x}_{\mathrm{g}}, \mathrm{y}_{\mathrm{g}}, \mathrm{z}_{\mathrm{g}}\right)=(0,0,0) \mathrm{m}$ in the inertial reference frame and the initial altitude is equal to zero $(\phi=\theta=\psi=0)$. $T_{R}$ and $T_{L}$ are assumed equal, and $\gamma_{R}=\gamma_{L}$. The simulation was run for 75 seconds starting at initial velocity $\mathrm{v}_{0}=2 \mathrm{~m} / \mathrm{s}$.

The INDI controller was evaluated in the presence of wind disturbances $\boldsymbol{v}_{\boldsymbol{w}}$, the model was simulated included a turbulent gust with an intensity of $2 \mathrm{~m} / \mathrm{s}$, in addition to a wind gust with gust amplitude of $2 \mathrm{~m} / \mathrm{s}$. This represents $33 \%$ of the maximum speed of the airship. The turbulence was generated by passing white noise through a filter to create Dryden model [23]. The sum vector of wind velocity $\boldsymbol{v}_{\boldsymbol{w}}$ was added to the velocity components in the $\mathrm{x}$ and $\mathrm{z}$ directions according to (5). Fig. 2 shows the tracking performance of the proposed controller and Fig. 3 shows the resulting pitch angle. The gondola's position with time is illustrated in Fig. 4. The results indicate the presence of small oscillations during horizontal flight and changes in altitude. The oscillation generated when the airship travels under influence of wind gust can be observed during longitudinal maneuvers. The control is able to limit these oscillations to $\pm 2.9^{\circ}$. The oscillations in Fig. 4 at $\mathrm{t}=7 \mathrm{~s}$ and $\mathrm{t}=62 \mathrm{~s}$ are due to the changing of the gondola's position to adjust the pitch angle in the windy condition. The tracking error of the altitude in the presence of wind is shown in Fig. 5.
The steady-state error is always produced when using only proportional control. Increasing or decreasing the proportional gain will result in a change in output however, changing the $K_{P}$ value allows for the rise time and steady state error to be reduced at the cost of increasing overshoot. Therefore, adding integral term has the effect of eliminating the steady-state error. However, it may have a negative effect on the transient response of the control system.

It can be seen in the result that during the airship ascent and descent period, the lift gas is unable to compensate the airship weight and follow the reference trajectory, therefore the thrust control will used to accelerate or decelerate airship up or down result in actuator saturation. In Fig. 2, the thrust components control is saturated when the rise of velocity and position control are required. The gondola actuator reaches the rate limit in the pitch angle control as observed in Fig. 4. The controller saturated during the ascent and reached its limit $s_{g}=-0.8 \mathrm{~m}$ and the controller was able follow the reference trajectory with no saturation during the descent.

The results show the robustness of the proposed control while subjected to aggressive manoeuvres and the airship remains stable even in the presence of unknown wind disturbance. The simulation results show that the displacement and the speed of the gondola are the primary factors affecting attitude control capability however, the moving-mass control is independent of velocity of the airship.

\section{CONCLUSION}

The incremental nonlinear dynamic inversion (INDI) approach was used for trajectory tracking control of an airship using a moving gondola and vectored thrust components as control inputs. The simulation results show that the proposed vehicle architecture allows for large pitch variations and rapid changes in altitude at any vehicle velocity. The vehicle was capable of producing changes in the vehicle's pitch of $80^{\circ}$ and $-88^{\circ}$ when the airship was ascending and descending, respectively. This configuration could potentially facilitate autonomous landings or payload deliveries in larger vehicles by ensuring that the gondola reaches the ground before the envelope in all vehicle orientations, and by minimizing the projected area of the lifting gas envelope near the gondola. The controller was able to track longitudinal motions with uncertain dynamics model. The results show that the robustness of INDI is sensitive to the quality of state measurements.

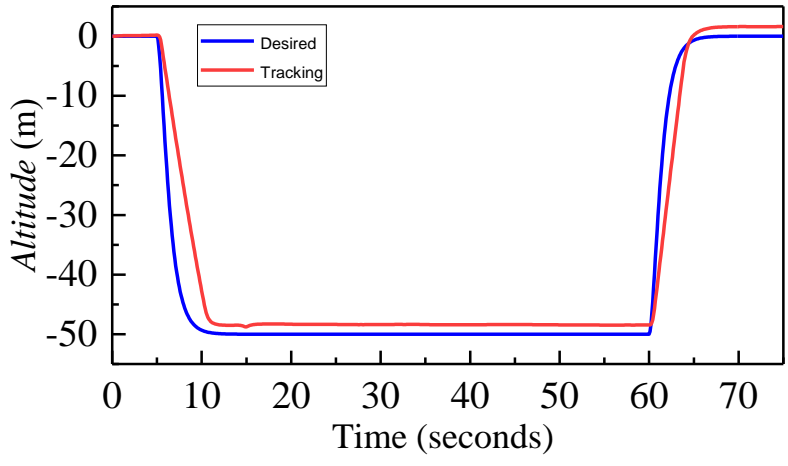

Figure 2. The reference trajectory and the tracking trajectory 


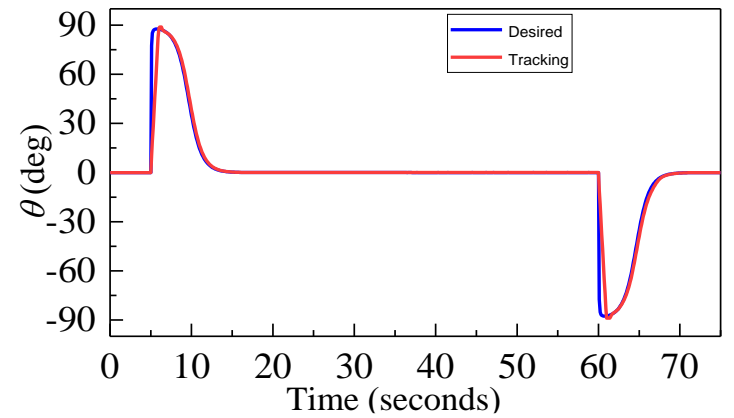

Figure 3. The reference pitch angle and simulated vehicle pitch

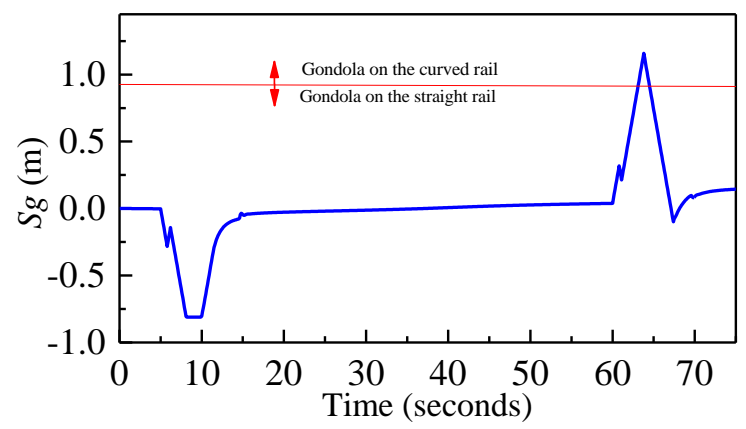

Figure 4. Gondola position
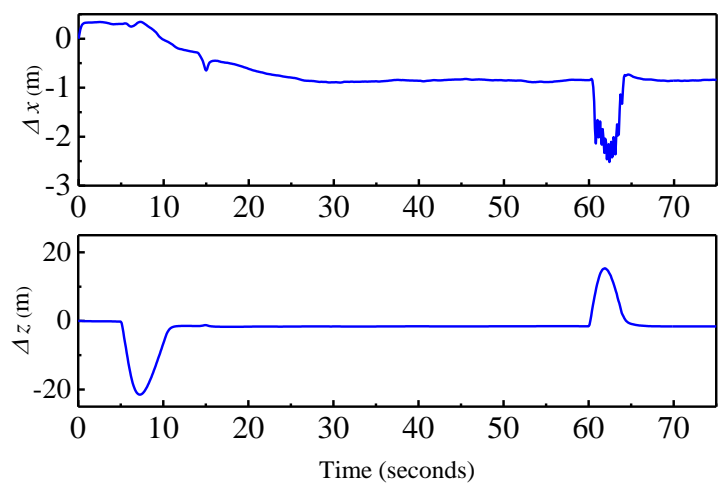

Figure 5. Tracking error

\section{REFERENCES}

[1] R. C. van't Veld, "Incremental Nonlinear Dynamic Inversion flight control," Delft University of Technology, 2016.

[2] B. Bacon and A. Ostroff, "Reconfigurable flight control using nonlinear dynamic inversion with a special accelerometer implementation," in AIAA Guidance, Navigation, and Control Conference and Exhibit, 2000, no. c, pp. 1-5.

[3] X. Wang, E. J. Van Kampen, Q. Chu, and P. Lu, "Stability analysis for incremental nonlinear dynamic inversion control," AIAA Guid. Navig. Control Conf. 2018, no. 210039, 2018.

[4] S. Sieberling, Q. P. Chu, and J. A. Mulder, "Robust flight control using incremental nonlinear dynamic inversion and angular acceleration prediction," J. Guid. Control. Dyn., vol. 33, no. 6, pp. 1732-1742, 2010.

[5] J. R. Azinheira, A. Moutinho, and J. R. Carvalho, "Lateral Control of Airship with Uncertain Dynamics using Incremental Nonlinear Dynamics Inversion," IFAC-PapersOnLine, vol. 48, no. 19, pp. 69-74, 2015.
[6] Z. Liu, J. Guo, M. Li, S. Tang, and X. Wang, "VTOL UAV Transition Maneuver Using Incremental Nonlinear Dynamic Inversion," Int. J. Aerosp. Eng., vol. 2018, pp. 1-19, 2018.

[7] E. Tal and S. Karaman, "Accurate Tracking of Aggressive Quadrotor Trajectories Using Incremental Nonlinear Dynamic Inversion and Differential Flatness," Proc. IEEE Conf. Decis. Control, vol. 2018Decem, pp. 4282-4288, 2019.

[8] P. Lu, E. J. van Kampen, C. de Visser, and Q. Chu, "Aircraft faulttolerant trajectory control using Incremental Nonlinear Dynamic Inversion," Control Eng. Pract., vol. 57, pp. 126-141, 2016.

[9] P. Smith and A. Berry, "Flight test experience of a non-linear dynamic inversion control law on the VAAC Harrier," in Atmospheric Flight Mechanics Conference, 2000, no. c, pp. 132-142.

[10] B. J. Bacon, A. J. Ostroff, and S. M. Joshi, "Reconfigurable NDI controller using inertial sensor failure detection \& isolation," IEEE Trans. Aerosp. Electron. Syst., vol. 37, no. 4, pp. 1373-1383, 2001.

[11] E. Lima and S. Da Silva, "Incremental Nonlinear Dynamic Inversion for Quadrotor Control," 2015.

[12] E. C. De Paiva, J. R. Azinheira, J. G. Ramos, A. Moutinho, and S. S. Bueno, "Project AURORA: Insfractructure and flight control experiments for a robotic airship," J. F. Robot., vol. 23, no. 3-4, pp. 201-222, 2006

[13] R. van 't Veld, E.-J. Van Kampen, and Q. P. Chu, "Stability and Robustness Analysis and Improvements for Incremental Nonlinear Dynamic Inversion Control,” no. January, 2018.

[14] P. B. Acquatella, W. Falkena, E. J. van Kampen, and Q. P. Chu, "Robust nonlinear spacecraft attitude control using incremental nonlinear dynamic inversion," AIAA Guid. Navig. Control Conf. 2012, no. August, 2012.

[15] Y. Huang, D. M. Pool, O. Stroosma, and Q. P. Chu, "Incremental Nonlinear Dynamic Inversion Control for Hydraulic Hexapod Flight Simulator Motion Systems," IFAC-PapersOnLine, vol. 50, no. 1, pp. 4294-4299, 2017.

[16] R. Coelho, "Quadrotor Attitude Control using Incremental Nonlinear Dynamics Inversion," TECNICO LISBOA, 2017.

[17] Z. Lu, P. Tang, and S. Zhang, "Incremental Nonlinear Dynamic Inversion Based Control Allocation Approach for a BWB UAV," no. August, 2018.

[18] C. Cakiroglu, E. J. Van Kampent, and Q. Chu, "Robust incremental nonlinear dynamic inversion control using angular accelerometer feedback," AIAA Guid. Navig. Control Conf. 2018, no. 210039, 2018.

[19] S. Gomes, "An Investigation of the Flight Dynamics of Airships with Application to the YEZ-2A." Cranfield Institute of Technology, p. 326, 1990.

[20] E. Lanteigne, W. Gueaieb, and R. S. Recoskie, "Unmanned Airship Design with Sliding Ballast: Modeling and Experimental Validation," 2016.

[21] H. Lamb, "The inertia coefficients of an ellipsoid moving in fluid," $\mathrm{Br}$. Aeronaut. Res. Commitee Rep. ..., no. 623, pp. 3-5, 1918.

[22] M. M. Munk, “Aerodynamics of airships, Aerodynamic Theory.” pp. $32-48,1936$.

[23] R. Beard and T. Princeton, Small Unmanned Aircraft: Theory and Practice, vol. 36, no. 1. 2013. 